J3eA, Journal sur l'enseignement des sciences et technologies de l'information et des systèmes,

Volume 3, Hors-Série 1, 5 (2004)

DOI : http://dx.doi.org/10.1051/bib-j3ea:2004605

(C) EDP Sciences, 2004

Perception de l'espace et locomotion des non-voyants par profilométrie laser : aides électroniques à la locomotion

\author{
R. Farcy ${ }^{1}$, R. Damaschini ${ }^{1}$, R. Legras ${ }^{1}$, R. Leroux ${ }^{1}$, \\ Y. Bellik ${ }^{2}$, C. Jacquet ${ }^{2}$, J. Greene $^{3}$, P. Pardo $^{3}$, \\ L. Agro ${ }^{4}$, J.M. Murienne ${ }^{4}$ et A. Zoghaghi ${ }^{4}$ \\ ${ }^{1}$ Laboratoire Aimé Cotton - CNRS, Bât. 505, F-91405 Orsay CEDEX, France \\ http://www.lac.u-psud.fr/ \\ ${ }^{2}$ LIMSI-CNRS, BP 133, F-91403 Orsay CEDEX, France \\ http:///www.limsi.fr \\ ${ }^{3}$ Association Valentin Haüy, 5 rue Duroc, F-75243 Paris CEDEX 07, France \\ ${ }^{4}$ Université Paris Sud, formation continue « aides optroniques à la locomotion », \\ Bât. 470, F-91405 Orsay CEDEX, France
}

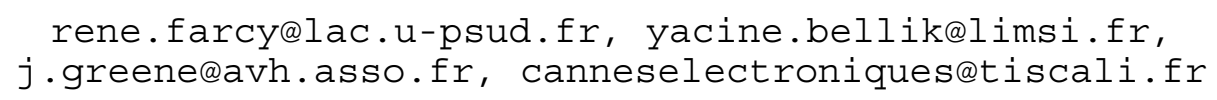




\title{
Perception de l'espace et locomotion des non-voyants par profilométrie laser : Aides électroniques à la locomotion
}

\author{
René Farcy ${ }^{1}$, Roland Damaschini ${ }^{1}$, Richard Legras ${ }^{1}$, Roger Leroux ${ }^{1}$ \\ Yacine Bellik $^{2}$, Christophe Jacquet ${ }^{2}$, Janet Greene ${ }^{3}$, Pierre Pardo ${ }^{3}$ \\ Laurence Agro ${ }^{4}$, Jean Marie Murienne ${ }^{4}$, Aziz Zoghaghi ${ }^{4}$ \\ ${ }^{1}$ Laboratoire Aimé Cotton - CNRS, Bat 505, 91405 Orsay cedex, \\ http://www.lac.u-psud.fr/ \\ ${ }^{2}$ LIMSI-CNRS, BP 133, 91403 Orsay cedex, http:///www.limsi.fr \\ ${ }^{3}$ Association Valentin Haüy, 5 rue Duroc 75243 Paris cedex 07 \\ ${ }^{4}$ Université Paris Sud, formation continue « aides optroniques à la locomotion », \\ Bat 470, 91405 Orsay cedex \\ rene.farcy@lac.u-psud.fr, yacine.bellik@limsi.fr, \\ j.greene@avh.asso.fr, canneselectroniques@tiscali.fr
}

\begin{abstract}
Résumé - Nous présentons les résultats en mobilité obtenus au quotidien par les non-voyants utilisateurs des dispositifs de profilométrie laser. Après un descriptif du contexte et des prérequis nécessaires pour aboutir à une utilisation performante des systèmes, nous en ferons une description sommaire, puis nous aborderons la démarche progressive d'apprentissage.
\end{abstract}

Mots clefs : handicap visuel, substitution sensorielle, profilométrie laser, locomotion.

\section{INTRODUCTION}

\section{A. Les objectifs}

Nous allons dans cette introduction insister sur le contexte d'utilisation des dispositifs «Tom Pouce» et « Télétact» utilisant l'optique active pour permettre aux non-voyants de percevoir l'espace, ceci afin d'éviter les interprétations naïves des résultats présentés. Notre objectif est de permettre aux non-voyants d'avoir une mobilité performante en toutes circonstances y compris dans les milieux dits hostiles : grands centres commerciaux, foules, milieux urbains très changeants, trajets inconnus pauvres en repères etc. La définition de la mobilité performante se réfère au comportement moyen de l'adulte voyant. Quatre conditions sont à satisfaire : l'autonomie dans la gestion du déplacement, la sécurité, la fluidité de la démarche, l'efficacité.

Un déficient visuel partant seul de chez lui et arrivant à destination en " piéton stop ", c'est à dire en sollicitant de multiples accompagnateurs sur son passage n'a pas une gestion autonome du trajet. Le fait de demander des renseignements aux passants en trajet inconnu est bien entendu compatible avec l'autonomie, c'est d'ailleurs ce que fait le voyant.

La sécurité dans le trajet implique de ne pas avoir sa survie dépendante des réflexes ultimes des automobilistes et des interventions spontanées de dernière seconde des passants.

La fluidité de la démarche peut s'évaluer en filmant la personne de dos et en compressant la vidéo à basse résolution, ce qui permet d'occulter la canne blanche. Une personne non-voyante ayant une très bonne fluidité ne sera pas discernable d'une personne voyante.

L'efficacité est le temps mis pour accomplir le trajet, devant être du même ordre de grandeur de celui d'un piéton adulte allant d'un pas moyen (ni pressé, ni flânant). Cette efficacité ne s'obtient pas en pressant le pas, mais en prenant de bonnes options et en ayant une démarche fluide.

Rappelons le temps mis par un enfant voyant pour obtenir une mobilité performante. La fluidité s'acquiert disons assez rapidement vers 3 ou quatre ans, quand l'enfant va seul anticiper longtemps à l'avance les obstacles, fonction de son objectif pour prendre au plus court. L'autonomie pour gérer un trajet de difficulté moyenne, être par exemple capable de trouver sa boutique et ses rayons préférés dans un grand centre commercial et de retrouver la voiture seul au parking va s'acquérir vers 6 ou 7 ans. La sécurité sur un trajet urbain nouveau, savoir où et quand traverser etc. ne sera acquise que vers 10 ans. Utiliser un plan pour faire un trajet nouveau n'est pas maîtrisé par tout les adultes voyants, c'est la question des nouveaux jeunes conducteurs : comment fait - on pour aller à un endroit que l'on ne connaît pas ?

Si nous insistons lourdement sur ces aspects évidents, c'est pour la raison suivante: sans la vue, tout est plus difficile, dans la mesure où la vue s'est adaptée aux repères naturels et que les repères urbains ont été « naturellement» pensé pour les voyants. Penser qu'il est possible de concevoir une machine miracle, dans l'état actuel de nos connaissances, qui va donner d'emblée à un non-voyant une mobilité performante relève d'une naïveté grave, malheureusement encore répandue y compris chez des chercheurs. Cela supposerait que l'on sache faire aussi bien que la vue en terme de capteurs et d'interface avec le cerveau, et que l'on disposerait d'injections intraveineuses vous permettant de maîtriser par exemple parfaitement le chinois au bout de quelques minutes.

\section{B. Les prérequis}

C'est donc pour ses raisons que nous allons insister sur les processus d'apprentissage et les pré-requis devant 
être en possession du non-voyant, aspects bien plus fondamentaux que les détails techniques des appareils, la personne étant le moteur essentiel de la performance et l'appareil un simple relais. En effet, indépendamment de toute technologie récente, il existe des méthodes permettant d'aller vers une mobilité de qualité pour un non-voyant dans de bonnes conditions de sécurité. Ces méthodes font appel à une autre façon d'exploiter les informations auditives, à une réorganisation des stratégies de déplacement, à l'utilisation de la canne blanche et à l'analyse des repères de rugosité au sol, à la connaissance non visuelle de l'urbanisation, au développement de méthodes mentales de représentation de l'espace etc. Il s'agit des techniques de locomotion enseignées depuis plus d'une trentaine d'années en France. Cet apprentissage du déplacement non visuel est long et cela est lié à l'acquisition de la masse de connaissances nécessaires, et au développement cognitif associé. Un appareil n'a pas pour vocation de se substituer à ce qui peut être fait naturellement et en serait d'ailleurs bien incapable. Nous considérons donc que la maîtrise de la locomotion classique est un pré-requis indispensable à l'utilisation d'un dispositif de haute technologie, tout comme elle l'est pour l'utilisation efficace d'un chien guide.

Un autre aspect essentiel est la motivation de la personne. Se déplacer de manière performante est difficile pour un non-voyant et il faut être franchement motivé pour l'autonomie. Nos maintenant cinq années d'expérience sur l'utilisation quotidienne des appareils nous ont amenés à ne pas croire non plus à l'effet miracle de la technologie sur la motivation des déficients visuels pour l'autonomie. Une personne réellement motivée pour l'autonomie aura déjà fait le maximum pour acquérir ce qui était possible avec les moyens existants et donc avec la «locomotion canne blanche ». On a observé de manière indéniable des surplus de motivation, mais toujours sur la base d'une autonomie préalable.

Notre objectif est d'améliorer la locomotion, en profitant de tous ses acquis et en repoussant ses limites, c'est pour cela que nous avons besoin de technologie. Nous recherchons l'équivalence avec le comportement du voyant en étendant les situations et contextes où cela devient possible. Un second axe important est d'arriver à rendre couramment accessibles ces performances y compris à ceux n'ayant pas forcément autant de facilités que les pionniers.

Nous allons maintenant faire un résumé des caractéristiques des deux appareils, puis décrire les étapes de l'apprentissage pour en faire une utilisation efficace.

\section{DESCRIPTION DES FONCTIONS DES APPAREILS ET APPRENTISSAGE}

\section{A.. Le "Tom Pouce»}

Nous disposons de deux appareils afin d'offrir une progression à l'utilisateur dans le processus d'apprentissage. Le premier appareil est un proximètre infrarouge. On pourra en trouver une description technique sommaire dans la référence [1]. Il détecte les obstacles à une portée au choix (interrupteur trois positions sous le pouce de l'utilisateur) de $0,5 \mathrm{~m} ; 1,5 \mathrm{~m}$ ou $3 \mathrm{~m}$. La protection en hauteur va du haut du genoux à la tête. La largeur angulaire latérale est de 20 degrés ce qui correspond à 1,5 mètres typiquement à la largeur des épaules et à trois mètres au double des épaules. L'information est transmise par une vibration du type tout ou rien. La vibration est fournie par le connecteur Jack situé en bas de l'appareil et arrive au niveau du petit doigt. La photographie qui suit représente l'appareil commercial de seconde génération. Il est prévu pour être en permanence associé à la canne. Ses deux piles AAA lui donnent une autonomie de plus de quarante heures de fonctionnement ce qui fait près d'un mois d'utilisation en moyenne. Tout débutant commence maintenant son apprentissage avec cet appareil.

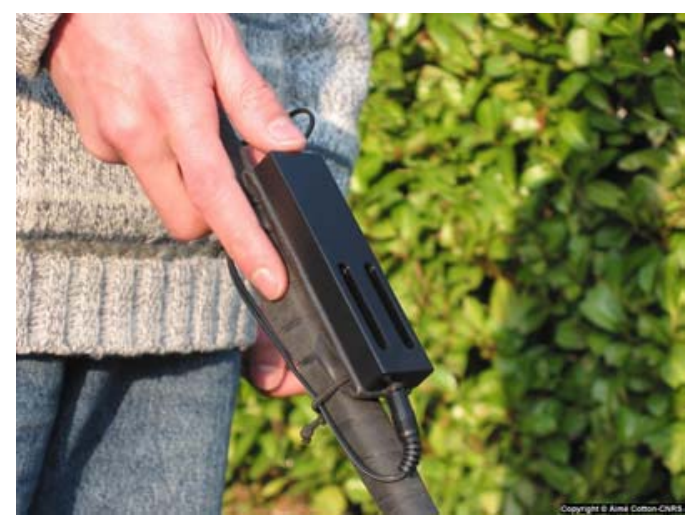

Fig.1. Photographie du Tom Pouce II.

La fonction fournie par l'appareil semble simple, l'utilisation ne l'est pas tant. En effet l'intérêt premier de l'appareil est l'anticipation à trois mètres de l'obstacle permettant, sur un trajet encombré, de trouver en permanence, dans la majorité des cas, le meilleur passage, celui du voyant. La canne étant virtuellement étendue à une longueur triple de l'habituel, la personne doit très bien centrer le pommeau de la canne au niveau du nombril et avoir une parfaite conscience de l'angle fait avec son axe corporel (le devant soi). Faute de quoi la personne croira trouver des obstacles sur sa trajectoire, qui sont en fait sur le côté, et n'évitera pas les obstacles frontaux. Il faut également que le débutant garde l'appareil dans la position verticale et ne fasse pas de rotations incontrôlées du pommeau de la canne. Ce centrage est déjà demandé en technique de canne blanche seule, mais son non respect n'a pas de conséquences aussi immédiates qu'avec le Tom Pouce, ne tolérant sur ce point aucun défaut technique.

Un second point est la mentalisation de l'anticipation à distance. Au début l'utilisateur à tendance à se comporter comme si l'obstacle ou le passage détecté à trois mètres se situait au bout de sa canne, ce qui amène à le contourner de manière trop brutale (défaut de fluidité) ou à mal localiser le passage (il tourne brutalement pour entrer dans un magasin et heurte la paroi avec la canne alors que l'entrée détectée sur le côté est encore à deux mètres). Il faut obliger l'utilisateur à utiliser en permanence la longue portée et le forcer à l'anticipation. Le réflexe spontané est de mettre la 
portée à 1,5 mètre et de réagir très rapidement comme si cela était un choc de la canne avec $50 \mathrm{~cm}$ de plus d'anticipation. Les bénéfices sont alors faibles.

$\mathrm{Si}$ le début du travail se fait dans des endroits relativement dégagés, on passe très vite à des foules denses, où il faut gérer les gens allant dans le même sens et à contre sens, ainsi que les obstacles fixes (y compris les gens arrêtés de dos ou de côté ne voyant pas le non-voyant arriver). Dans ce contexte une très bonne perception de la direction de la canne est importante, car l'appareil émet de manière saccadée des vibrations, il faut avoir bien conscience de ce qui correspond à des passants latéraux non dangereux et frontaux dangereux. Il faut savoir si la personne arrive de face en vous voyant et vous évitant, ou est distraite, si elle est arrêtée...Si elle vient de vous doubler et vous bouche le passage... Il semble bizarre d'arriver à gérer tout ces cas avec une information aussi sommaire. Cela est pourtant possible et s'est avéré très reproductible sur plusieurs dizaines d'utilisateurs suivis en formation. On procède de la façon suivante : l'enseignant (obligatoirement un instructeur de locomotion expérimenté dans l'enseignement de la canne blanche, ayant suivi un stage sur l'utilisation des appareils), fait des mises en situations où il prévient qu'il va jouer le rôle d'un voyant de face allant éviter le non-voyant (celui - ci ne doit pas alors modifier son rythme de marche). Il reprend l'exercice en se comportant comme un passant le doublant, puis joue le passant arrêté regardant une vitrine (le non-voyant doit alors contourner), puis finalement le passant distrait lui fonçant dessus (le non-voyant doit marquer un temps d'arrêt attirant l'attention du voyant et lui laissant le temps de se ressaisir). Une fois les cas analysés séparément, faisant appel au recoupement de la durée des vibrations, de la direction de la canne, de l'écoute auditive des éventuels bruits de pas, on lance la personne dans une foule réelle. Généralement cela débute par un excès de prudence, mais qui donne le bénéfice immédiat de ne plus avoir les pieds des passants qui se prennent constamment dans la canne. Les nombreuses erreurs d'attention des voyants sont compensées par les petits ralentissements ou temps d'arrêt de l'utilisateur laissant au voyant le temps d'esquiver la canne. Petit à petit l'utilisateur arrive à prendre de la vitesse et à déambuler de manière tout à fait normale dans ce contexte.

Il est très important que l'enseignement soit fait par un professionnel bien au fait des consignes de sécurité pour les déplacements en situation de déficit visuel. Une demande ou reproche fait à l'appareil est qu'il ne détecte pas les bordures de quelques centimètres de haut, pouvant être une source de croche patte. Une technique correcte de balayage glisser de la canne blanche permet de régler le problème en locomotion classique. Un appareil détectant ces bordures interpréterait une remontée de trottoir comme un obstacle et induirait un comportement dangereux en fin de traversée de route. On apprend d'ailleurs en formation qu'un obstacle non détecté par l'appareil et produisant un choc canne a une très forte probabilité d'être une marche ou un trottoir à franchir (le contexte faisant le reste).

Pour une personne ayant une bonne base préalable de déplacement à la canne l'enseignement prend entre 10 et 20 séances étalées sur 3 à 4 mois. En début d'apprentissage toute la concentration de la personne est placée sur les informations de l'appareil. Peu à peu elle apprend à hiérarchiser l'information parmi les autres informations sensorielles, et à l'intégrer de manière réflexe. Il faut alors attendre quelques mois pour que le temps total de réaction incluant la décision et le comportement moteur associé descende à la fraction de seconde ce qui permet l'utilisation optimale des fonctions. Nous n'entrerons pas ici dans les techniques fines d'utilisation amenées par les premiers bons utilisateurs, permettant d'aller un peu au delà des fonctions prévues de l'appareil.

Il s'agit d'un appareil débutant aux performances limitées, en effet l'information en tout ou rien de résolution angulaire limitée (20 degrés) n'apporte pas d'information précise sur le relief de la configuration. La portée est limitée à trois mètres, il n'est pas possible d'offrir plus dans la mesure où avec 20 degrés d'ouverture, l'appareil ne trouverait plus les passages de taille humaine à distance plus grande. Les petits poteaux fin noirs ne sont pas détectés avant la canne et les grands panneaux de circulation cataphotes sont détectés trop en avance. Ce sont des limitations inhérentes aux techniques de proximétrie infrarouge utilisées, ayant par ailleurs de bon côtés (simplicité de mise en œuvre, bonne immunité à la lumière diurne et à la pluie etc.). L'utilisateur réussissant la formation (un sur deux environ à l'heure actuelle, plus si la sélection est sévère) obtient un gain de fluidité, une réduction du stress et un confort dans le déplacement. Par contre la fluidité et l'anticipation dans le déplacement ne peuvent s'assimiler à une démarche de voyant que dans les environnements suffisamment dégagés et ne présentant pas de configurations imprévues complexes. Un piéton voyant commence à réagir à plus de trois mètres à un obstacle, et la différence se sent dans la trajectoire plus courbe et moins centrée sur le passage de l'utilisateur du Tom Pouce même aguerri.

Le bon utilisateur est souvent sceptique sur l'obtention d'un service supérieur par un autre appareil. Nous proposons en moyenne à une personne sur deux ayant franchi cette étape (les plus à l'aise) de passer à la suivante : le Télétact.

\section{B. Le « Télétact»}

Il s'agit d'un télémètre laser ayant une vingtaine de mètres de portée, couplé à un proximètre infrarouge ainsi qu'à divers autres capteurs destinés à supprimer les biais de mesure de la télémétrie laser. On pourra trouver des détails techniques sur les principes techniques de fonctionnement dans les références [2] et [3]. L'important est que l'information obtenue par les capteurs permet d'obtenir dans la quasi totalité des configurations une mesure correcte de la distance de l'obstacle rencontré par le faisceau laser indépendamment de sa nature (poteau noir fin, panneau cataphote... en toute condition de luminosité, de l'obscurité totale au plein soleil). La figure suivante présente une photo de la seconde génération commerciale des appareils clippé de manière facilement amovible sur la canne. 


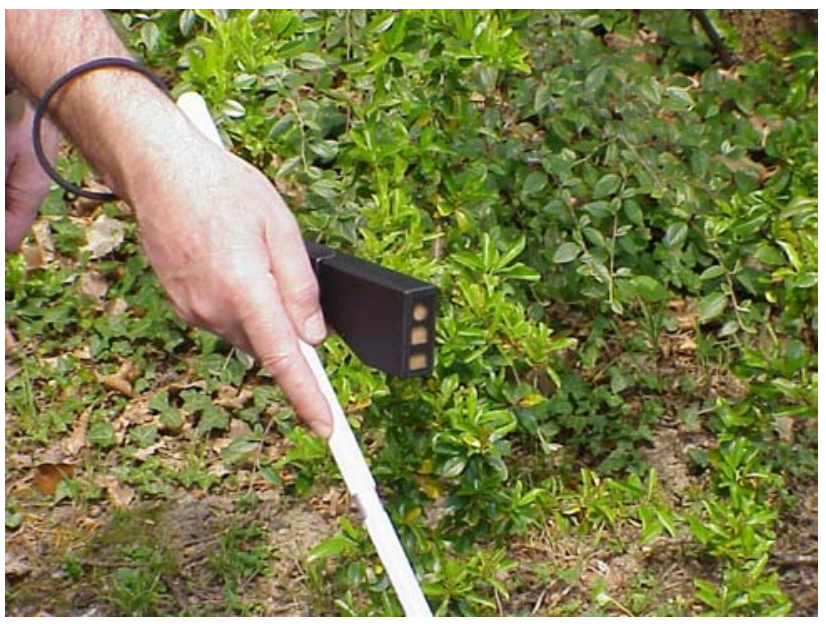

Fig.2. Photographie du Télétact II.

Il y a deux différences essentielles avec le Tom Pouce, la portée qui peut aller jusqu'à vingt mètres et la finesse angulaire, puisque l'on bénéficie de la finesse du faisceau laser. L'information sur la distance est transmise soit par voie sonore (28 notes de musiques allant de l'aiguë (courtes distances) au grave (longues distances)), soit par voie tactile (trois niveaux de distances, 0-1,5m, 1,5m-3m, 3m-6m répartis sur deux vibreurs). On trouvera une description détaillée des interfaces dans la référence [4]. La personne, en balayant sa canne, perçoit un véritable profil de l'environnement dans le plan horizontal. Afin de permettre à l'utilisateur de progresser dans la continuité, après le Tom pouce on lui propose le Télétact en version tactile clippé systématiquement sur la canne comme sur la photo. La transition est assez indolore (tout en demandant à nouveau de ré entrer dans un processus de concentration intense). La personne apprécie au bout de quelques heures les bénéfices de l'anticipation accrue à 6 mètres ainsi que la précision angulaire avec laquelle les obstacles sont perçus. Un peu plus tard, la possibilité d'avoir une information grossière du relief commence à être exploitée. Les exercices se font de manière privilégiée en centre commercial aux heures de pointe, là où la personne est amenée à gérer le plus d'imprévus en un minimum de temps. Toutes les aptitudes développées pour le Tom Pouce sont ré exploitées avec une finesse accrue. Il semble que le plus dur reste les fonctions de base au Tom Pouce et que le gain en finesse par le Télétact tactile entre dans la logique d'un utilisateur averti, même si cela lui demande de nouveaux efforts non négligeables. Il faut que l'instructeur soit exigeant en demandant la prise en compte de l'obstacle à 6 mètres dans le comportement et pas seulement à trois mètres comme le fait spontanément l'ancien utilisateur du Tom Pouce. A ce stade l'utilisateur a rapidement la curiosité de vouloir éprouver les sensations d'un découpage en plus d'intervalles et d'une portée de 20 mètres. Nous ne savons à ce jour transmettre cette information que de manière sonore. Il faut donc entrer dans un nouveau processus cognitif. Ce n'est pas «le son» en soi qui est gênant, mais la quantité d'information présente qu'il va falloir apprendre à filtrer. Cette transition qui reste difficile pour tout le monde est souvent motivée par la conviction de la personne qu'elle arrivera après à faire encore plus de choses par ce moyen. L'un des apprentissages essentiels au Télétact sonore (valable dans une moindre mesure pour la version tactile) est la mentalisation des distances et des directions absolues. On pointe l'appareil sur un obstacle, on prend son profil par un léger balayage, on éteint l'appareil et on va toucher l'obstacle. Au début on rate l'obstacle car on passe légèrement à côté, on sous estime ou surestime la distance. L'important est d'être capable de se mentaliser l'obstacle qui a généré des sons comme si on avait ouvert momentanément les yeux. Cet obstacle est en tête et même si on s'est déplacé, on est capable de revenir dessus à tout moment sans réutiliser l'information de l'appareil. Un des autres exercices de base consiste à repérer deux obstacles d'un point donné et d'aller faire un huit entre eux, appareil éteint.

Une autre fonction difficile à maîtriser, mais utile est la perception précise des reliefs latéraux en mouvement de marche. Suivre une personne se faufilant dans la foule se fait très bien, mais n'est pas accessible au débutant. Il faut taper régulièrement avec le faisceau laser sur l'un des côtés de la personne, en maintenant une note de musique fixe et en marchant dans sa trajectoire. Dès que la personne dévie, on sent dans le poignet que le son s'est déplacé et il faut aussitôt asservir sa propre trajectoire.

Pour augmenter les possibilités de perception et bénéficier d'une réelle perception à trois dimensions, il faut prendre l'appareil à la main en le déclippant de la canne. La proprioception de la personne (aptitude à percevoir l'orientation de ses membres grâce aux articulations) donne deux angles, le télémètre laser la distance, ce qui permet une profilométrie 3D complète. Maintenant l'orientation manuelle du faisceau de haut en bas avec 20 mètres de portée n'est pas une chose facile, contrairement à l'orientation droite gauche qui s'acquiert rapidement. En effet sur le plan cognitif que ce soit visuellement, auditivement ou par la proprioception, la conscience du haut bas est bien moins développée que la conscience du droite gauche chez l'homme. Afin de garantir la meilleure interprétation des données, il est recommandé d'utiliser sa main principale (la droite pour les droitiers) et de reléguer la canne dans l'autre main.

A ce stade, les meilleurs utilisateurs ont eu parfois grand peine à convaincre de leur cécité. En trajet connu, même complexe, le test du film de dos pour la fluidité ne pose pas de problèmes, et l'efficacité du déplacement est tout à fait comparable au voyant. En milieu inconnu, si les informations de repères préalablement fournies sont bonnes cela se passe bien. La personne est également apte à aborder de manière rationnelle un centre commercial inconnu.

Les vidéos des exercices de base et des tests de performance peuvent être consultées sur le site www.lac.upsud.fr. Il faut prévoir un temps additif de formation supérieur à celui du Tom Pouce pour arriver à une utilisation de haut niveau du Télétact. 


\section{CONCLUSION}

Les plus de dix ans de travail sur la technologie des capteurs et les cinq années de travail en locomotion et sur l'utilisation effective au quotidien des appareils nous ont amenés à certaines convictions. Ces techniques d'optique active font bon ménage avec la canne blanche et les deux sont indispensables à une mobilité très performante. Le rôle de l'utilisateur est absolument primordial, un bon utilisateur à la canne seule fera mieux qu'un mauvais utilisateur avec le meilleur des «Télétact». Afin de rendre impossible cette situation, les appareils ont été exclu de la vente libre. Ils ne sont accessibles que suite à une formation réussie, cette formation étant financée par le Lions Club international. Durant l'année scolaire 2002-2003, 40 personnes sont entrées dans le processus de formation. 24 en sont sorties avec un succès au Tom pouce, et 8 l'ont transformé en succès Télétact. Nous espérons doubler les entrants l'année prochaine et accroître le taux de réussite. Les appareils seront améliorés dans la continuité. Les développements nouveaux s'orientent sur un véritable système de localisation inertiel prolongeant l'information obtenue en espace dégagé à l'extérieur par le GPS. Nous voulons obtenir un fonctionnement dans les intérieurs, y compris les métros, sans équipement spécifique des infrastructures. Une seule chose est sûre pour les nouveaux développements, les bases de locomotion classique seront toujours des pré-requis aussi primordiaux.

\section{REFERENCES}

[1] Guidance - Assist systems for the blind, René Farcy, Roland Damaschini, EBIOS 2000, Amsterdam, 3-5 Juillet 2000.

[2] Triangulating laser profilometer as navigational aid for the blind: Optical aspects, R. Farcy, B. Denise, R. Damaschini, Applied Optics, 1 march 1996, vol. 35, nº 1161-1166.

[3] Triangulating laser profilometer as three-dimensional space perception system for the blind, R. Farcy, R. Damaschini, Applied Optics, 1 November 1997, vol. 36, $\mathrm{n}^{\circ} 31, \mathrm{p}$ 8227-8232.

[4] Locomotion Assistance for the Blind, R. Farcy, Y. Bellik, Universal Access and Assistive Technology, S. Keates, P. Langdon, P.J. Clarkson, P. Robinson (Eds.), Springer, ISBN 1-85233-595-5, 2002. 